\title{
Electronic and optical properties of Si and Ge nanocrystals: An ab initio study
}

\author{
Olivia Pulci ${ }^{\mathrm{a}}$, Elena Degoli ${ }^{\mathrm{b}, *}$, Federico Iori $^{\mathrm{c}}$, Margherita Marsili ${ }^{\mathrm{a}}$, \\ Maurizia Palummo ${ }^{\text {a }}$, Rodolfo Del Sole ${ }^{\mathrm{a}}$, Stefano Ossicini ${ }^{\mathrm{b}}$ \\ ${ }^{a}$ Dipartimento di Fisica dell'Università di Roma Tor Vergata, European Theoretical Spectroscopy Facility (ETSF), CNR-INFM-SMC, \\ NAST, via della Ricerca Scientifica, I-00133 Roma, Italy \\ ${ }^{\mathrm{b}}$ CNR-INFM-S ${ }^{3}$ and Dipartimento di Scienze e Metodi dell'Ingegneria, Università di Modena e Reggio Emilia, via Amendola 2 Pad, \\ Morselli, I-42100 Reggio Emilia, Italy \\ ${ }^{\mathrm{c}}$ LSI, Ecole Polytechnique, Palaiseau, France
}

\section{A R T I C L E I N F O}

\section{Article history:}

Available online 17 July 2009

\section{Keywords:}

Silicon

Germanium

Nanocrystals

Optical properties

MBPT

\begin{abstract}
A B S T R A C T
First-principles calculations within density functional theory and many-body perturbation theory have been carried out in order to investigate the structural, electronic and optical properties of undoped and doped silicon nanostructures. We consider Si nanoclusters co-doped with $B$ and P. We find that the electronic band gap is reduced with respect to that of the undoped crystals, suggesting the possibility of impurity based engineering of electronic and optical properties of Si nanocrystals. Finally, motivated by recent suggestions concerning the chance of exploiting Ge dots for photovoltaic nanodevices, we present calculations of the electronic and optical properties of a $\mathrm{Ge}_{35} \mathrm{H}_{36}$ nanocrystal, and compare the results with those for the corresponding $\mathrm{Si}_{35} \mathrm{H}_{36}$ nanocrystals and the co-doped $\mathrm{Si}_{33} \mathrm{BPH}_{36}$.
\end{abstract}

(c) 2009 Elsevier Ltd. All rights reserved.

\section{Introduction}

In recent years the studies of semiconductor quantum structures, such as silicon and germanium nanocrystals (nc), have raised hopes for their actual use in the photovoltaic and photonic fields. In fact, nanocrystalline Si shows optical gain in a variety of situations [1] and could hence be used, in the future, as an optically active material. Moreover, innovative photovoltaic devices can take advantage of quantum confinement in both silicon and germanium. Nanocrystals, in particular, provide the opportunity to control the energy of carrier states by adjusting the confinements in all three spatial

\footnotetext{
* Corresponding author. Tel.: +39059 2055289.

E-mail address: elena.degoli@unimore.it (E. Degoli).
} 
dimensions, resulting in effective band gap energies well above the bulk band gap, tunable using the nc diameter. Moreover, as experimentally shown by Fujii and co-workers [2] the gap tuning can also be obtained by co-doping of nanomaterials with n-type and p-type impurities like boron and phosphorus. The development of advanced devices based on Si and Ge nc structures requires the theoretical investigation and the understanding of their electronic and, in particular, optical properties.

In this paper we present a first-principles study of the optical properties, beyond the density functional theory, of Si and Ge based nc, focusing on the role played by the material, by confinement, and by co-doping with boron and phosphorus impurities. We fix our attention on a spherical nc composed of 35 atoms whose surface is fully saturated with $\mathrm{H}$. The comparison will then be done between $\mathrm{Si}_{35} \mathrm{H}_{36}, \mathrm{Ge}_{35} \mathrm{H}_{36}$ and $\mathrm{Si}_{33} \mathrm{BPH}_{36}$ optical spectra.

\section{Theoretical method}

Density functional theory (DFT) [3] is powerful for studying ground state properties of materials, and represents the starting point of several excited state methods. DFT enables one to treat systems of hundreds of atoms, and is here used to calculate the formation energy of doped and co-doped Si nc.

Optical properties of nc are here computed in the framework of many-body perturbation theory (MBPT) within the so called Bethe-Salpeter equation. In particular, MBPT takes into account the excitonic effects (namely the effect of the interaction between the electron and hole formed by the optical excitation), solving an effective two-body Hamiltonian, called the excitonic Hamiltonian [4]. Ingredients of this approach are the DFT wavefunctions and the GW [4] single-quasi-particle eigenvalues. The macroscopic dielectric function is then calculated using the eigenvalues and eigenvectors of the excitonic Hamiltonian.

\section{Doping Si nc}

Fujii et al. [2] have experimentally shown that doping control at the nanoscale can be used to modify electronic and optical properties of Si nc. It is hence important to study in detail from first principles the effect of doping on the electronic structure and on the absorption and emission spectra. Here, we consider B and P co-doped Si nc that, as already shown [5], are more stable with respect to single P or B doped nc, thanks to charge compensation. Moreover, as shown in Fig. 1(a), sub-surface second-neighbor configurations for $\mathrm{P}$ and $\mathrm{B}$ impurities present a negative formation energy (defined as the energy for inserting the $B$ and $P$ dopants, after extracting two Si atoms from the nc) with respect to the case in which $B$ and $P$ are located at the largest possible reciprocal distance. This demonstrates that a stronger interaction between impurities favors co-doping. Keeping the distance between the impurities (as sub-surface second neighbors) fixed then, we can compare the HOMO-LUMO gaps (calculated at the DFT level) of several co-doped and undoped Si nc. As shown in Fig. 1(b) we find that, in the presence of co-doping, the Si nc gaps are strongly reduced with respect to that of the undoped cases, showing the possibility of impurity based engineering of the optical properties of Si nc.

\section{Optical spectra}

The calculated absorption optical spectrum of $\mathrm{Si}_{33} \mathrm{BPH}_{36}$ is shown in Fig. 2(a). For comparison, we also show the optical spectra of the undoped nc (Fig. 2(b)). Moreover, encouraged by recent measurements which demonstrate that Ge nanocrystals act as photocurrent generators in an electrochemical cell [6], hence offering the chance of exploiting Ge dots for photovoltaic nanodevice use, we have calculated the optical properties of $\mathrm{Ge}_{35} \mathrm{H}_{36}$ nc (Fig. 2(c)).

We see that the onsets of absorption in the three cases are quite similar, in agreement with Reboredo and Zunger [7]. The main differences among the three spectra come from the absorption strength: below $5.5 \mathrm{eV}$, the absolute value is larger for Ge than for Si nc, the doped Si nc being the one with the lower intensity.

A complete study of $\mathrm{Ge}$ and $\mathrm{Si}$ nc has been done in [8] within the TDLDA. Our spectra are in qualitative agreement with those of [8] apart from the energy positions: our GW + BSE spectra are blueshifted with respect to the TDLDA ones. Differences between BSE results and TDLDA ones are also 

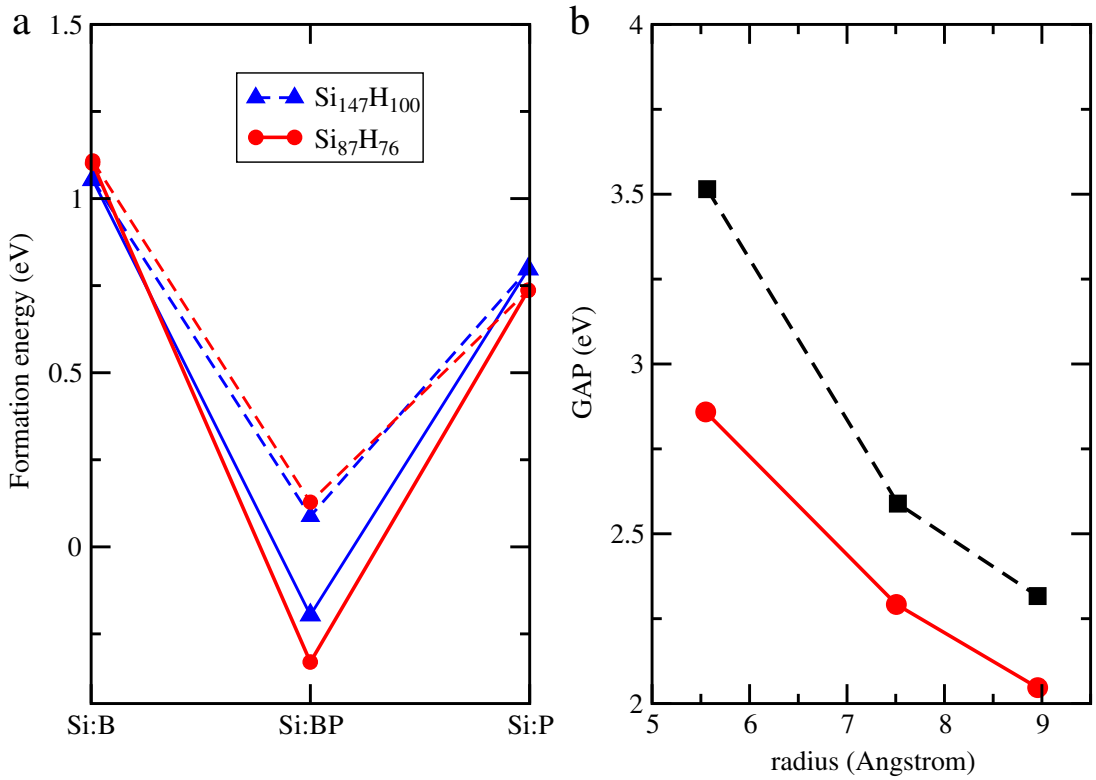

Fig. 1. (a) Formation energy for single and co-doped Si nc. Two different impurity-impurity distances in the co-doped nanocrystals are considered (dashed and solid lines, large and small distances, respectively; see the text). The lines are a guide for the eyes. (b) DFT HOMO-LUMO gaps for undoped (squares) and co-doped (circles) Si nc, for several sizes. The lines are a guide for the eyes.
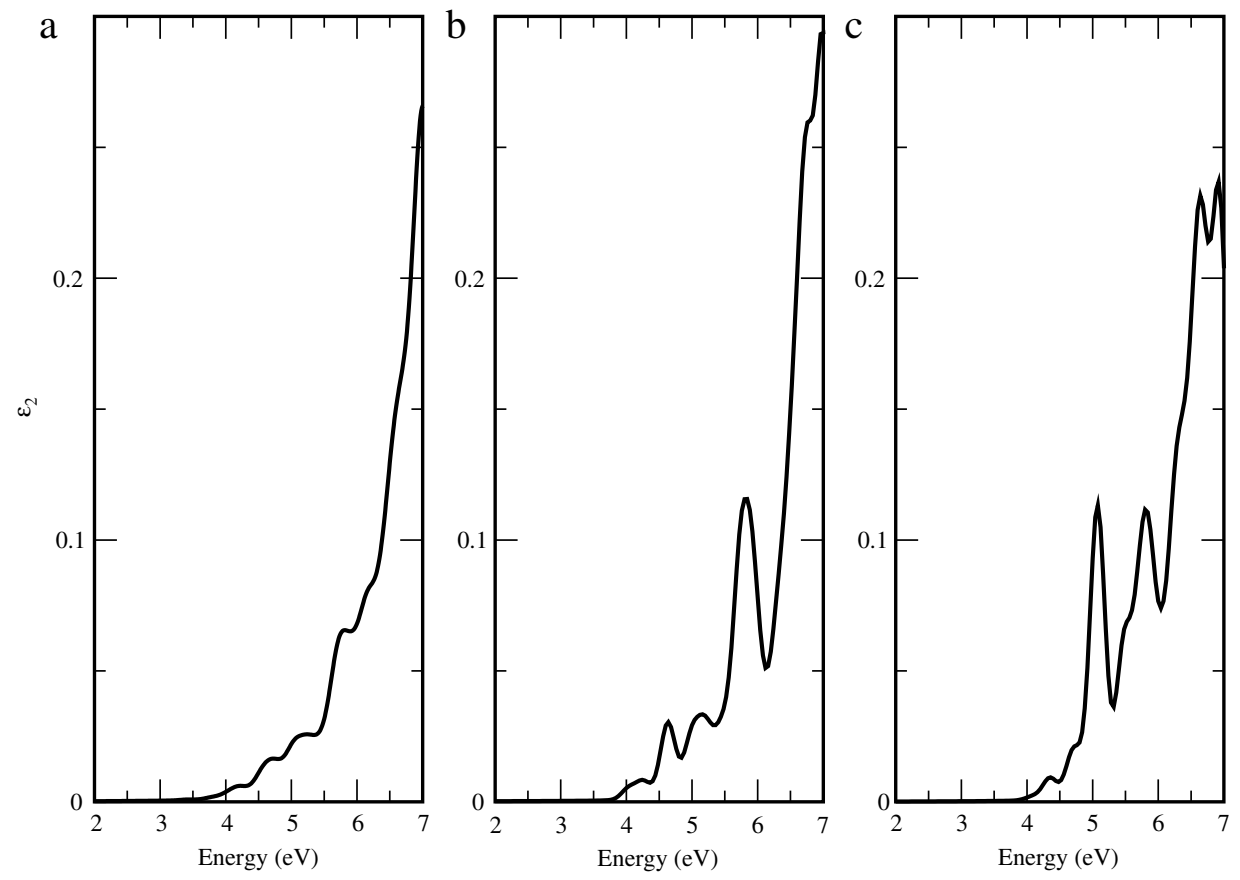

Fig. 2. Imaginary part of the dielectric function, of $\mathrm{Si}_{33} \mathrm{BPH}_{36}$ (a), $\mathrm{Si}_{35} \mathrm{H}_{36}$ (b), and $\mathrm{Ge}_{35} \mathrm{H}_{36}$ (c), normalized to a volume of $40 \times 40 \times 40 \mathrm{au}^{3}$. Many-body effects are included within the GW + BSE approach. 
present for other isolated systems such as the CdSe clusters studied in [9] by del Puerto et al. and in the optical properties of indole molecules in solution [10]. These differences can be traced back to the presence and the limitations of the various approximations used in the two theoretical approaches.

\section{Conclusion}

The optical properties of a 35-atom semiconductor nc have been studied in the framework of many-body perturbation theory, showing the possibility of tailoring its optical properties not only by changing the nc size but also by playing with the material ( $\mathrm{Si}$ or $\mathrm{Ge}$ ) and doping.

\section{References}

[1] L. Pavesi, L. Dal Negro, C. Mazzoleni, G. Franzó, F. Priolo, Nature 408 (2000) 440;

L. Dal Negro, et al., Appl. Phys. Lett. 82 (2003) 4636;

M. Cazzanelli, D. Kovalev, L. Dal Negro, Z. Gaburro, L. Pavesi, Phys. Rev. Lett. 93 (2004) 207402;

K. Luterová, et al., Appl. Phys. Lett. 84 (2004) 3280.

[2] M. Fujii, Y. Yamaguchi, Y. Takase, K. Ninomiya, S. Hayashi, Appl. Phys. Lett. 87 (2005) 211919.

[3] P. Hohenberg, W. Kohn, Phys. Rev. 136 (1964) B864;

W. Kohn, L.J. Sham, Phys. Rev. 140 (1965) A1113.

[4] for a review see for example G. Onida, L. Reining, A. Rubio, Rev. Modern Phys. 74 (2002) 601.

[5] F. Iori, et al., Phys. Rev. B 76 (2007) 085302.

[6] M. Scarselli, et al., Appl. Phys. Lett. 91 (2007) 141117;

M. De Crescenzi et al. Superlattices and Microstructures (in print).

[7] F.A. Reboredo, A. Zunger, Phys. Rev. B 62 (2000) R2275.

[8] A. Tsolakidis, R. Martin, Phys. Rev. B 71 (2005) 125319.

[9] M.L. del Puerto, M.L. Tiago, J.R. Chelikowsky, Phys. Rev. B 77 (2008) 045404.

[10] A. Mosca Conte, E. Ippoliti, R. Del Sole, P. Carloni, O. Pulci, J. Chem. Theory Comput. 5 (2009) 1822. 